WORLD

PSYCHIATRIC ASSOCIATION

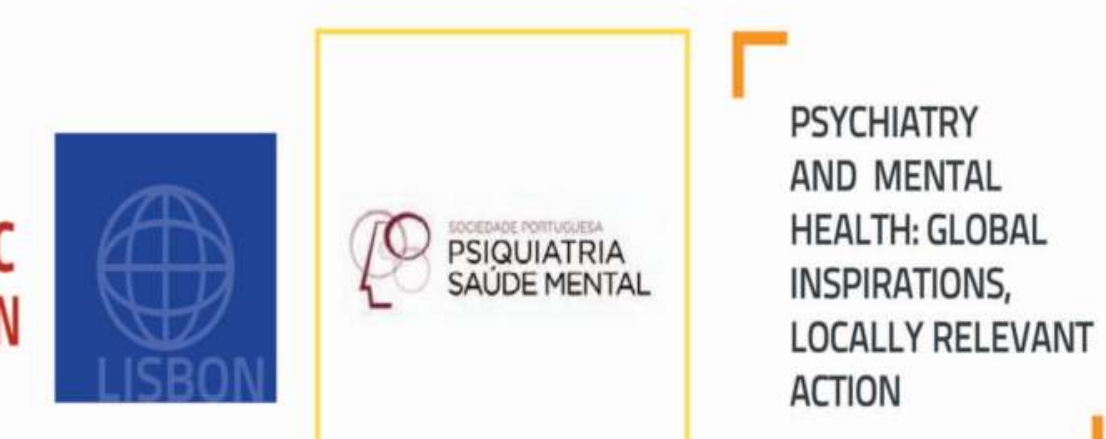

\title{
A Cross Sectional Study of Anxiety Disorders in Medical School Students
}

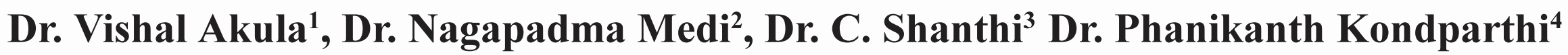 \\ ${ }^{1}$ Associate Professor Osmaina Medical College Hyderabad, \\ ${ }^{2}$ Consultant Anaesthesiologist Indur Multi Speciality and Neuro Psychiatric Hospital, Nizamabad, India \\ ${ }^{3}$ Associate Professor Govt Medical College, Nizamabad, India \\ ${ }^{4}$ Asst. Professor, Osmania Medical College, Hyderabad, India
}

\section{Objectives:}

1) To study State and Trait anxiety in medical school students.

2) To study the year of education and State and Trait anxiety in medical school students.

3) To Study the correlation between socio demographic variable and anxiety in medical school students.

\section{Background:}

Compared to general population medical school students are showing higher levels of anxiety. So, this study was conducted to evaluate anxiety disorders in medical school students and its association with socio demographic variables.

Materials and Methods:

A cross-sectional study with a convenient sample of $(n=210)$ medical school students at sub urban medical school was performed. State Trait Anxiety Inventory (STAI) was used to measure anxiety symptoms and the person's vulnerability to develop anxiety symptoms and their associations with socio demographic were evaluated. Modified Kuppuswamy Scale was used to measure socioeconomic status.

\section{Results}

Medical students having anxiety symptoms on STAI - State anxiety $10 \%$ low severity, $64.3 \%$ medium and $25.7 \%$ high severity. STAl -Trait anxiety scores were $10 \%$ low, $57.6 \%$ medium and $32.4 \%$ high scores. $59 \%$ of the sample belongs to sub urban area which is statistically significant ( $P$ value $<0.001)$ and majority were first year medical students $(41.9 \%)$ which is also statistically significant $(P$ value $<0$. 001). Year of education showing significant levels of trait anxiety symptoms among medical school students.

\section{Conclusions:}

Medical school students are have medium levels of anxiety symptoms, male gender have increased levels of anxiety symptoms compared to females. First year medical students have increase in State anxiety symptoms and Second year medical students have high Trait anxiety symptoms.

\section{Keywords:}

Medical students, state anxiety, trait anxiety 